\title{
Escenario catastrófico y utópico de muertes maternas, Danlí, Honduras, 2018 - 2030
}

\author{
Catastrophic and utopian scenarios of maternal deaths, Danlí, Honduras, 2018-2030 \\ Lorenzo Ubaldo Pavón Rodríguez, ${ }^{1}$ \\ Ángel Rene Estrada Arévalo. ${ }^{2}$
}

\begin{abstract}
${ }^{1}$ Médico General, Máster en Administración de la salud, Máster en Salud Pública, Tegucigalpa, Honduras; ${ }^{2} \mathrm{PhD}$, Doctor en Gobierno y Administración Pública; Universidad Nacional Autónoma de Chiapas UNCH, Chiapas, México.
\end{abstract}

\begin{abstract}
RESUMEN. Antecedentes: El Gobierno de Honduras transcribió su compromiso de cumplir con los Objetivos de Desarrollo Sostenible (ODS) para el año 2030. En el ODS 3 se propone llegar a una tasa de 70 muertes maternas a nivel nacional por cada 100 mil nacidos vivos. Del 2006 al 2017, el sistema de información sanitaria en Danlí, El Paraíso, captó 41 muertes maternas. El cumplimiento de los ODS significaría que para el año 2030 esto representaría 129 muertes maternas distribuidas en los 18 departamentos de Honduras. Objetivo: Construir escenarios para la mortalidad materna en el municipio de Danlí, Honduras, para el período 2018 2030. Metodología: Estudio cualitativo y cuantitativo. Se construyeron escenarios catastrófico y utópico utilizando el método de prospectiva y la técnica de grupo focal. Participantes en el grupo focal incluyeron representantes de la Secretaría de Salud y de la comunidad. Todos los participantes proporcionaron consentimiento informado verbal. Resultados: Los resultados revelaron que en los próximos 13 años en el Municipio de Danlí se podrán presentar un escenario catastrófico de 115 muertes maternas o un escenario utópico de 34 muertes maternas. Discusión: Los resultados sugieren un escenario catastrófico con tendencia ascendente de muertes maternas de no haber intervenciones. El escenario utópico será descendente si se realizan intervenciones eficaces en salud.
\end{abstract}

Palabras clave: Desarrollo sostenible, Interpretación estadística de datos, Mortalidad materna.

\section{INTRODUCCIÓN}

Según la Organización Mundial de la Salud (OMS), la razón de mortalidad materna en Honduras para los años 1990, 1995, 2000, 2010 y 2015, fueron 272, 166, 150, 155 y 129 por 100,000 nacidos vivos, ${ }^{1}$ logrando un cambio porcentual de la razón entre los años de 1990 y 2015 de 52.6. La OMS reportó que en Honduras la tasa de mortalidad materna para el año 2010 fue 73 por 100,000 nacidos vivos, sensiblemente menor a la reportada anteriormente. ${ }^{2}$ Sin embargo, en este mismo estudio se señala que los departamentos de Gracias a Dios, Islas de la Bahía, El Paraíso, La Paz e Intibucá, presentaron tasas de 201, 159, 141,128 y 113 por cada 100,000 nacidos vivos, respectivamente. Honduras, aunque presentó progreso, no logro alcanzar la meta de los Objetivos de Desarrollo del Milenio (ODM) y como país presentó importantes brechas tanto al interior de sus departamentos como en sus municipios, por lo que refleja un gravísimo problema del sistema de salud hondureño.

El Gobierno de Honduras transcribió su compromiso de cumplir con los Objetivos de Desarrollo Sostenible (ODS) para el $2030 .{ }^{3}$ En el Objetivo 3 se propone llegar a una tasa de 70 muertes maternas a nivel nacional por cada 100 mil nacidos vivos. Del

Recibido 11-10-2018; Aceptado para publicación 8-2-2019

Dirección para correspondencia: Dr. Lorenzo Pavón

Correo electrónico: msc.Ipavon@gmail.com

Conflictos de interés. Los autores no tienen conflictos de interés que declarar con respecto a este artículo.

DOI: https://doi.org/10.5377//rmh.v87i1.11929
2006 al 2015, el sistema de información sanitaria en el municipio de Danlí, El Paraíso, captó 41 muertes maternas. Ante el anuncio, del cumplimiento de los ODS, significa que para el año 2030 esto representaría 129 muertes maternas distribuidas en los 18 departamentos del país, con un total de 7 casos por Departamento, lo que indica a nivel municipal aproximadamente 1 muerte materna para una población de 184,033 nacidos vivos año 2017 según cifras del Registro Nacional de las Personas. ${ }^{4}$

La importancia para el Gobierno de Honduras y los gerentes en salud de estimar lo que sucederá con el indicador de mortalidad materna al 2030, año en que Honduras será evaluada bajo el marco de las políticas sanitarias de los ODS, radica en poder implementar acciones que mejoren los resultados. Ante este marco de políticas sanitarias se justifica los compromisos nacionales con el ODS - 3 sobre salud y bienestar, relacionados con las metas de cobertura universal de salud y reducción de la mortalidad materna. El presente estudio se realizó con el objetivo de construir escenarios catastrófico y utópico de muertes maternas en el municipio de Danlí, Honduras, para el período 2018 - 2030, con el propósito de contribuir a la toma de decisiones que permitan reducir la mortalidad materna en Honduras.

\section{METODOLOGÍA}

Estudio cualitativo con un componente cuantitativo. Para la construcción de escenarios se utilizó el método de la prospectiva como un panorama de los futuros posibles de un sistema destinado a iluminar las consecuencias de las estrategias de 
acción proyectadas.$^{5-7}$ La prospectiva como metodología científica tiene como objeto principal la creación de propuestas 0 situaciones diversas que podría suceder en el sector de la salud proyectadas como escenarios a futuro. Cada escenario construido (catastrófico y utópico) plantea un nivel de razón para la toma de desiciones en el sector de salud, planificando a largo plazo con el fin de que la alta gerencia cuente con toda la información pertinente en relación a este trazador y en miras a los ODS. Se utilizó la técnica de los grupos focales para definir los escenarios.

El universo estuvo constituido por 41 muertes maternas totales registradas desde el 2006 al 2017 por la Región Departamental de Salud de El Paraíso (ver Cuadro 1). De abril - diciembre 2017, se revisó el registro de casos anuales en los consolidados de muertes de mujeres en edad fértil y muertes maternas (10 - 49 años) de la Región Departamental de El Paraíso desde el año enero 2006 - diciembre 2017 (serie de tiempos del pasado). La unidad de análisis fueron las muertes maternas, en el municipio de Danlí, en el periodo 2006 - diciembre 2017.

El estudio se desarrolló en tres fases. La Fase 1, incluyó el análisis de los datos de las muertes maternas de manera exploratoria, enero 2006 - diciembre 2017. La Fase 2 fue cuantitativa; se desarrolló la proyección de tendencias del pasado, presente y futuro - forecast o pronóstico al $2030 .{ }^{8}$ El método estadístico utilizado fue tendencia lineal, el análisis de los datos se basó en el programa QM - Excel. La Fase 3 fue cualitativa: la conformación del grupo focal, como técnica utilizada para el taller de prospectiva en salud. El grupo focal fue integrado por un conjunto heterogéneo conformado por personas incluyendo personal de salud, y de la comunidad, entre ellos dos pediatras, dos epidemiólogos, tres salubristas, un médico general, dos auxiliares y una Licenciadas en Enfermería, un paramédico del Cuerpo de Bomberos, un fiscal de la niñez y dos fiscales de mujer y el resto (6) personas de la comunidad. La contrucción de los escenarios planteados fue realizada por consenso del grupo focal. En la Figura 1 se presenta la lógica de construcción de mapa de escenarios de prospectiva en salud para la mortalidad materna en el municipio de Danlí, Honduras, período 20182030. Las preguntas al cuestionario aplicado al grupo focal fueron validadas por tres jueces expertos resultando un alfa de Cronbach: 97.34, Guttman: 90 y Spearman: 0.95. Se planteó la hipótesis de trabajo de que la tendencia de los dos escenarios de muertes maternas es ascendente a partir del 2018 a 2030.

Se contó con el aval de las autoridades de la Región Departamental de Salud de El Paraíso, sede Danlí, para la realización de este estudio. Los participantes fueron invitados a participar mediante consentimiento informado verbal, explicando el objetivo del estudio. Se les explicó que su participación era voluntaria.

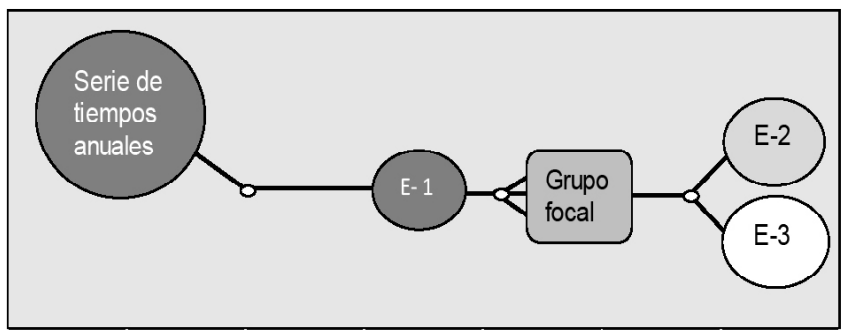

Figura 1. Lógica de construcción de mapa de escenarios de prospectiva en salud para la mortalidad materna en el municipio de Danlí, Honduras, período 2018-2030. Serie de tiempos anuales representa todos los casos de muertes maternas del período 2006-2017. E-1, Escenario Inercial (tendencia): representa el número de casos anuales de forma ascendente o descendente. E-2, Escenario catastrófico: representa escenario en futuro con aumento de muertes maternas anuales. E- 3, Escenario utópico: representa escenario en futuro con disminución de muertes maternas anuales.

\section{RESULTADOS}

El escenario inercial de la mortalidad materna de la serie de tiempo de doce años fueron 41 casos evidenciados a partir de enero 2006 a diciembre del año 2017, de esa serie de tiempo se encontró un valor mínimo de 1 muerte materna para el año 2007 y un máximo de 10 para el año 2017, registradas en todo el municipio de Danlí. Con una tasa de variación absoluta porcentual de muertes maternas de: 233\% en relación al año 2017 vs. 2016 (ver Cuadro 1).

El escenario catastrófico para la mortalidad materna fue construido en una serie de trece años a partir el 2018 al 2030, los actores sociales que formaron el grupo focal, determinaron un total de 115 casos para el período de trece años, con un mínimo de seis muertes (años 2018, 2021 y 2022) y un máximo de trece muertes (2028), en el municipio de Danlí (ver Figura 2).

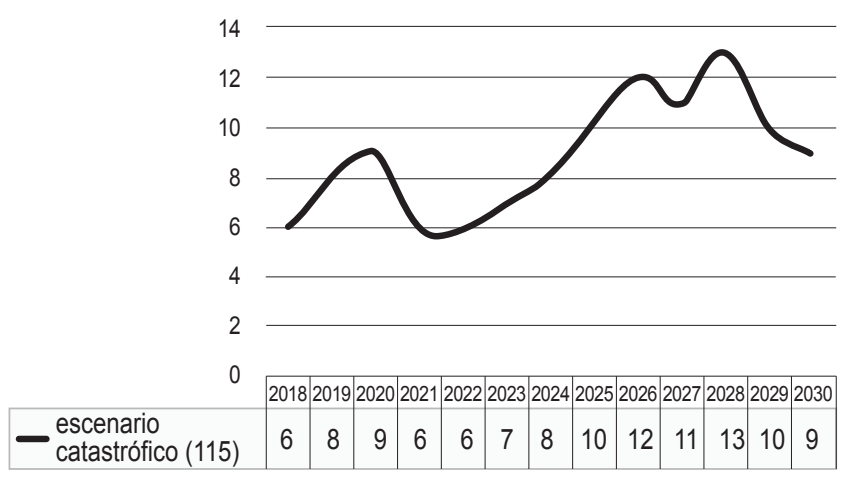

Figura 2. Escenario catastrófico de muertes maternas en el municipio de Danlí, Honduras, período 2018-2030, construido con la información brindada por el Grupo Focal. ODS=Objetivos de Desarrollo Sostenible.

Cuadro 1. Serie de tiempo anual de muertes maternas en el municipio de Danlí, El Paraíso, Honduras, período 2006-2017.*

\begin{tabular}{lcccccccccccc}
\hline Años & 2006 & 2007 & 2008 & 2009 & 2010 & 2011 & 2012 & 2013 & 2014 & 2015 & 2016 & 2017 \\
\hline Muertes maternas (número) & 2 & 1 & 4 & $\mathrm{~s} / \mathrm{r}$ & 6 & 2 & 2 & 7 & $\mathrm{~s} / \mathrm{r}$ & 4 & 3 & 10 \\
\hline
\end{tabular}

S/r= sin registro. *Fuente de información: Secretaría de Salud de Honduras. Dirección General de Vigilancia de la Salud, Programa de Atención a la Mujer, Región Departamental de Salud de El Paraíso, consolidado de muertes en edad fértil y muertes maternas (10-49 años). 


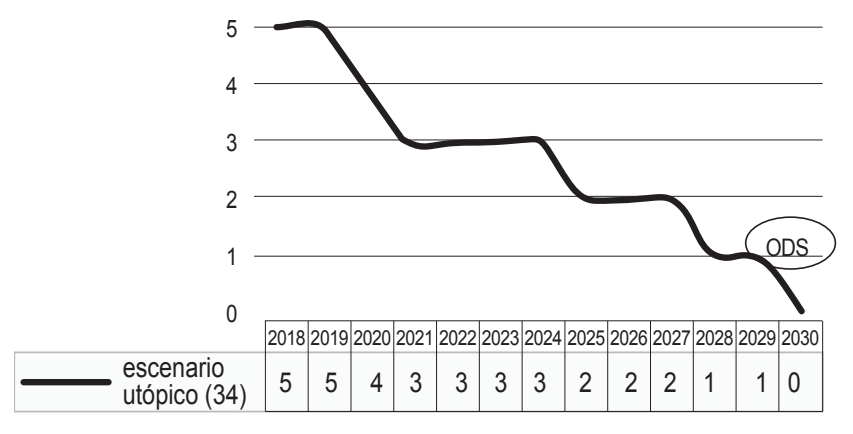

Figura 3. Escenario utópico de muertes maternas en el municipio de Danlí, Honduras, período 2018-2030, construido con la información brindada por el Grupo Focal. ODS=Objetivos de Desarrollo Sostenible.

Respecto a la construcción del escenario utópico para el mismo trazador humano sanitario, los actores sociales determinaron un total de 34 casos para el período 2018 al 2030, con un mínimo de cero (año 2030) y un máximo de cinco (años 2018 y 2019) muertes para todo el municipio de Danlí (ver Figura 3).

\section{DISCUSIÓN}

Se pudo construir los escenarios catastrófico y utópico para el indicador de salud planteado. El escenario catastrófico para la mortalidad materna en el municipio de Danlí posiblemente será ascendente como se indica para los próximos años hasta el año 2030, lo que indica que posiblemente habrá un total de 115 defunciones maternas a partir del 2018 al 2030 en otras palabras, se presentarán al menos nueve (9) casos de muertes maternas por año en dicho municipio. Estos datos indican que si no se realizan intervenciones y que si las cosas siguen pasando como lo últimos años el escenario catastrófico demostrará que Honduras no cumplirá con los ODS en especial con la reducción de la mortalidad materna. El escenario utópico fue determinando como descendente a partir del mismo período antes mencionado. Posiblemente en este escenario solo se presentarán 34 muertes maternas, lo que significa que de existir intervenciones oportunas o formulación de políticas públicas micro regionales en salud se presentarían 3 casos al año hasta lograr la evaluación de los ODS.

El mayor impacto de las muertes maternas en el municipio de Danlí estará representado por la construcción del escenario catastrófico..$^{8-10}$ La diferencia para la mortalidad materna en relación con su pasado es que seguirá posiblemente un escenario catastrófico ascendente y para la otra dimensión del escenario utópico, posiblemente sea un escenario descendente pero aun manteniendo cifra críticas y devastadoras para el sistema de salud hondureño. El nivel inaceptable de mortalidad materna prevenible es un desafío a nivel mundial, pese a los significativos progresos realizados en los últimos años; el riesgo de fallecer sigue desproporcionadamente más elevado entre los segmentos más vulnerables de la sociedad y los esfuerzos deben encaminarse a la reducción de las desigualdades y la discriminación, mejorar la asistencia que se presta a mujeres y niños incluyendo ámbitos de salud sexual y reproductiva. Entre las estrategias debe tomarse en cuenta las pautas cambiantes de la fertilidad y las causas de muerte también cambiantes, aun con tasas de mortalidad materna en disminución, fenómeno conocido como "transición obstétrica". ${ }^{11}$ Por lo tanto, es sumamente necesario seguir investigando y dar seguimiento a este trazador sanitario, y expandirlo a nivel departamental, como un modelo de prospectiva en salud, sin olvidar toda su metodología y su aplicación en este caso. En este marco es importante la creación o formulación y evaluación de las nuevas políticas sanitarias municipales con vísperas a los ODS y determinadas a que el escenario catastrófico no llegue a impactar en el proceso de salud enfermedad- y riesgo como ocurrencia de muerte en la población de mujeres en edad fértil. El presente estudio se limitó a la construcción de escenarios de las muertes maternas anuales a partir del período encontrado, faltando la cuantificación de los años 2000 al 2005.

La construcción de escenarios en prospectiva, ${ }^{12}$ nos permite anticiparnos a los acontecimientos que pueden suceder en un futuro, sumado a la mejora del indicador de mortalidad materna en el municipio de Danlí. Para Godet, ${ }^{12}$ la prospectiva es un panorama de los futuros posibles de un sistema destinado a iluminar las consecuencias de las estrategias de acción proyectadas, para Miklos, ${ }^{13}$ Garanda \& Osorio ${ }^{14}$ es la ciencia que construye escenarios a futuro basados en experiencias pasadas para anticiparnos a los posibles acontecimientos y abordar mejor los problemas relacionados con la salud de las comunidades. Se utilizaron las explicaciones y orientaciones brindadas por Estrada R. y Rocha J. en una entrevista presentada por el Centro de Investigaciones y Estudios de la Salud (CIES), Nicaragua, sobre cómo construir los escenarios catastrófico y utópico a partir del inercial (tendencial) y catastrófico y utópico usando la prospectiva y grupos focales. Se utilizó la técnica de los grupos focales con aplicación de la herramienta de cuestionario tal como lo propone Miklos que facilitó la construcción de los escenarios en salud con la aplicación de un instrumento validado, tal como lo proponen Miklos, ${ }^{13}$ Garanda \& Osorio, ${ }^{14}$ Fossati, ${ }^{15}$ de León, ${ }^{16}$ Tobar. ${ }^{17}$ Partiendo del marco de las caracteristicas que deben tener los escenarios como ser multiples (dos o mas), con análisis de fuerzas sociales con vinculos de decisiones estratégicas, estimaciones de supuestos para correr modeles cuantitativos de pronóstico y con acontecimietos políticos, sociales y ambientales y económico. Con ello permite el análisis de riesgo y vulnerabilidad a largo plazo, ${ }^{18}$ tal como se ha planteado en este estudio basado a largo plazo 2030 con actores claves para la anticipación y resolver los problemas de salud de las embarazdas a nivel municipal.

Frente a esta antesala, tanto la comunidad, el Gobierno municipal, el Estado de Honduras y sus altas autoridades sanitarias deben mirar hacia el futuro y cumplir con la reducción de la mortalidad materna como este trazado para cumplir con uno de los ODS construyendo un escenario utópico (deseable) tal como lo promueven otros investidaores ${ }^{18} \mathrm{y}$ poder garantizar una mejor salud materna, como también nombrar a Danlí el primer municipio saludable en Honduras en estos conceptos. 


\section{CONTRIBUCIONES}

LUPR y AREA participaron en todas las fases del estudio, desde su concepción hasta el análisis. LUPR lideró el desarrollo del artículo y ambos autores aprobaron la versión final del mismo. Esta investigación, es producto del protocolo de tesis doctoral: Formulación de política pública para la cobertura uni- versal de la salud en el municipio de Danlí, Honduras en el marco del derecho a la salud 2015 - 2018.

\section{AGRADECIMIENTO}

A la Región Departamental de Salud de El Paraíso, sede Danlí, por su apoyo al facilitar la información para el análisis central de este estudio.

\section{REFERENCIAS}

1. Organización Mundial de la Salud. Evolución de la mortalidad materna 1990-2015 estimaciones de la OMS, el UNICEF, el UNFPA, el Grupo del Banco Mundial y la División de Población de las Naciones Unidas. Sinopsis [internet]. Ginebra: OMS; 2015. [consultado 12 mayo de 2018]. Disponible en: http://apps.who.int/iris/bitstream/10665/204114/1/WHO_ RHR 15.23_spa.

2. Organización Mundial de la Salud. Salud en las Américas: Honduras-Mortalidad. [Internet]. Washington D.C.: OMS; 2012. [consultado 23 de abril de 2017]. Disponible en: http://www.paho.org/salud-en-las-americas-2012/ index.php?option=com_content\&view=article\&id=43:honduras\&ltemid $=16$ 0\&lang=es

3. Nacionaes Unidas. Objetivos de desarrollo sostenible [Internet]. New York: ONU; 2015. [consultado 22 de Agosto de 2018].Disponible en: https:// www.un.org/sustainabledevelopment/es/objetivos-de-desarrollo-sostenible/

4. Registro Nacional de las Personas(HN). Datos estadísticos de inscripciones 2017: por Departamentos y genero 2017 [Internet]. Tegucigalpa: RNP; 2018. [consultado 18 septiembre de 2018]. Disponible en: http://www.rnp. hn/wp-content/uploads/2018/08/Inscripciones-2017.pdf

5. Rodríguez CM. Pensamiento prospectivo: visión sistémica de la construcción del futuro. Análisis [internet].2014 [consultado 22 de Agosto de 2018];46(84): 89-104. Disponible en: http://dx.doi.org/10.15332/s01208454.2014.0084.05

6. Rivera FJU. Análisis estratégico y prospectivo en salud: el enfoque de Godet y simulaciones de escenarios para el Programa de Salud Familiar (PSF). En: Análisis estratégico en salud y gestión a través de la escucha [Internet]. Rio de Janeiro: FIOCRUZ; 2006, pp. 153-186. Disponible en: 10.747/9788575415429.

7. Miklos T, Jiménez E, Arroyo M. Prospectiva, gobernabilidad y riesgo político instrumentos para la acción. Mexico D.F: LIMUSA; 2008.

8. Bas E. Tipos de predicción. En: Prospectiva cómo usar el pensamiento sobre el futuro. Que es y que no es, la Prospectiva. $3^{\text {a }}$. Ed. Barcelona: Ariel; 2010. p 25-35.

9. Antezana P. Desafíos para el fortalecimiento de la democracia y la gobernabilidad democrática en América Latina: el análisis político y la construcción de escenarios prospectivos - PAPEP [Internet]. New York: Escuela
PNUD; 2010. [consultado 8 de septiembre de 2017].Disponible en: https:// lideresdeizquierdaprd.files.wordpress.com/2015/11/desafc3ados-parael-fortalecimiento-de-la-democracia-y-la-gobernabilidad-en-amc3a9ricalatina.pdf

10. García Sánchez E, Valencia Velazco ML. Planeación Estratégica. Técnica y práctica. $2^{\mathrm{a}}$. ed. Mexico: Trillas; 2012. P81-88.

11. Organización Mundial de la Salud. Estrategias para poner fin a la mortalidad materna prevenible [internet]. Washington D.C.: OMS;2014. [consultado 8 de septiembre de 2018]. Disponible en: https://www.who.int/reproductivehealth/topics/maternal perinatal/spanish epmm.pdf

12. Miklos T, Tello M. Planeación prospectiva: una estrategia para el diseño del futuro. México: LIMUSA; 2017. p.162, 177.

13. Miklos T, Arroyo M. Prospectiva y escenarios para el cambio social [internet]. Mexico: UNAM; 2008. [consultado 4 de marzo de 2018]. Disponible en: https://madrid.tomalaplaza.net/files/2011/07/WORKING_PAPERS_8. pdf

14. Gándara G, Osorio Vera F. Métodos Prospectivos manual para el estudio y construcción del futuro. México: Paidos; 2014.

15. Fossati CE, Paiva de Lima ML, Volkmer de Azambuja AM. Prospección de escenarios relacionados a la construcción de los puertos uruguayos en la región de la Laguna Merín. J. Transp [internet].2016[consultado 6 de septiembre de 2017];10(1):5-9. Disponible en: http://www.scielo.br/scielo. php?script=sci_arttext\&pid=S2238-10312016000100005.

16. de Leon Ardon RV. Construcción de scenarios [internet]. Mexico: Universidad Nacional Autónoma de México;2017. [consultado 8 de septiembre de 2017]. Disponible en: http://www.ingenieria.unam.mx/sistemas/PDF/ Avisos/Seminarios/Seminario\%20III/2\%20Construccion_Escenarios.pdf

17. Tobar F. Análisis de tendencias y construcción de escenarios [internet]. Buenos Aires: UBA; 2004. [consultado 8 de septiembre de 2017]. Disponible en: https://studylib.es/doc/4632394/anlisis-de-tendencias-y-construccin-de-escenarios.

18. Asociación Nacional de Facultades y Escuelas de Ingeniería (MX). Ingeniería industrial en México 2030: escenarios del futuro, estudio de planeación prospectiva. México: ANFEl; 2010.

ABSTRACT. Background: The Government of Honduras transcribed its commitment to comply with the Sustainable Development Goals (SDG) for year 2030. The SDG 3 proposes to reach a rate of 70 maternal deaths at national level for every 100 thousand live births. From 2006 to 2017, the health information system in Danlí, El Paraíso, captured 41 maternal deaths. Compliance with the SDGs means that by the year 2030 this would represent 129 maternal deaths distributed in the 18 departments of Honduras. Objective: To build maternal mortality scenarios at Danli District, Honduras, for the period 2018-2030. Methodology: Qualitative and quantitative study. We build catastrophic and utopic scenarios using the prospective method and the focal group technique. Focal group participants included representatives from the Ministry of Health and the community. All participants provided verbal informed consent. Results: The analyzed results revealed that during the next 13 years in Danli district could possible occur a catastrophic scenario of 115 maternal deaths or a utopian scenario of 34 maternal deaths. Discussion: The catastrophic scenario suggests that this will be ascending only if interventions are not made. The utopian scenario will be descendant if effective health interventions are carried out.

Keywords: Data interpretation, statistical; Maternal mortality; Sustainable development. 